\title{
Editorial
}

\section{Partially Connected and Automated Traffic Operations in Road Transportation}

\author{
Md Abdus Samad Kamal $\mathbb{D}^{1},{ }^{1}$ Mohsen Ramezani $\mathbb{D}^{2},{ }^{2}$ Guoyuan Wu, ${ }^{3}$ Claudio Roncoli $\mathbb{D}^{4},{ }^{4}$ \\ Jackeline Rios-Torres $\left(1,{ }^{5}\right.$ and Olivier Orfila $\mathbb{1}^{6}$ \\ ${ }^{1}$ Graduate School of Science and Technology, Gunma University, 1-5-1 Tenjincho, Kiryu 376-8515, Japan \\ ${ }^{2}$ The University of Sydney, School of Civil Engineering, Sydney, NSW, Australia \\ ${ }^{3}$ Department of Electrical and Computer Engineering, and Center for Environmental Research and Technology, UC Riverside, \\ Riverside, CA, USA \\ ${ }^{4}$ School of Engineering, Aalto University, Espoo 02150, Finland \\ ${ }^{5}$ Energy and Transportation Science Division, Oak Ridge National Laboratory, Oak Ridge, TN 37831, USA \\ ${ }^{6}$ IFSTTAR, 25 allée des Marronniers, 78000 Versailles, France
}

Correspondence should be addressed to Md Abdus Samad Kamal; maskamal@ieee.org

Received 28 October 2019; Accepted 29 October 2019; Published 15 January 2020

Copyright (C) 2020 Md Abdus Samad Kamal et al. This is an open access article distributed under the Creative Commons Attribution License, which permits unrestricted use, distribution, and reproduction in any medium, provided the original work is properly cited.

Transportation is a key driver of development, enabling people to have access to jobs, education, health, and goods. Still, the unintended consequences of transportation constitute a big risk, threatening the global sustainable development. About $64 \%$ of global oil consumption and $23 \%$ of the worldwide $\mathrm{CO}_{2}$ emissions are attributed to transportation [1]. Moreover, every year, congestion accounts for billions of dollars due to wasted time and fuel consumption, and the World Health Organization (WHO) [2] estimated that 1.3 million people died on roads in 2015. Connected and Automated Vehicles (CAVs) hold the potential to improve the current operational safety and efficiency of the transportation system by relieving drivers from some or all the driving tasks and enabling the cooperation among vehicles, between vehicles, and roadway infrastructure or other road users.

A variety of CAV applications have been devised, modeled, simulated, and deployed to substantialize their performance recently. They mostly target various traffic scenarios and contexts including traffic coordination at intersections and merging, dynamic speed control on the highway, traffic forecasting, and anticipative vehicle control. These research efforts have revealed the benefits of CAVs, including the potential to improve safety [3], alleviate traffic congestion [4] and reduce fuel consumption and emissions $[5,6]$.

With the full penetration of CAVs, the traffic can be operated in a fully automated manner achieving undoubtedly improved traffic performances. However, many challenges remain before a massive deployment of CAVs can be witnessed. It is then expected that CAVs will gradually appear in the market, increasing the complexity of the current transportation system as vehicles with different levels of connectivity and automation will start interacting with manually driven vehicles. There have been some early efforts to explore the implications of these complex interactions [7-10]. Overall, these early attempts seem to show a consensus on the benefits of higher market penetrations of vehicles enabled with connectivity and/or automation, but there is still a large level of uncertainty regarding the effects of lower market penetrations. Furthermore, a priori prediction of interaction between human and CAVs, now relying on simulation methodologies needs new numerical developments in order to be more representative of the reality. The design of prospective scenarios is also a challenge while trying to predict the impact of a system that will not be widespread in the market before several decades.

The ongoing and future research will probably provide more insights and innovative frameworks for analysis. This special issue includes research and review articles focusing on the connectivity in vehicular traffic with the presence of autonomous vehicles for potential improvement in traffic performances. It covers seven novel research articles including one review describing the modeling of traffic with CAVs, and use of CAVs for traffic light control and monitoring the traffic network, which are summarized as follows: 


\section{Modeling Traffic with CAVs}

Review Article: Simulation-Based Connected and Automated Vehicle Models on Highway Sections: A Literature Review.

In this paper, Wooseok et al. conducted a detailed review on modeling methods of vehicle motion that are used in recent simulation-based studies of the intelligent vehicles (CAVs with various levels of autonomy), and provided new insights for future intelligent vehicle analyses, potential scopes, and research gaps. The connectivity of vehicles is essential to improve the roadway capacity by the automated vehicles (AVs). However, the improvements in the roadway capacity and energy consumption in traffic directly depend on the market-penetration rate of intelligent vehicles. The necessity of experimental calibration of models recently developed for AVs is highlighted, and the comprehensive study of the socio-economic impact of such vehicles has not been conducted. With respect to the car-following and lane-changing characteristics of intelligent vehicles, empirical data are needed for the model calibration.

Article: Modeling Microscopic Car-Following Strategy of Mixed Traffic to Identify Optimal Platoon Configurations for Multiobjective Decision-Making.

In this paper, a naïve strategy for microscopic car-following in a mixed traffic scenario has been proposed. Depending on the driving system of consecutive vehicles, a vehicle with an automated driving system (ADS) may decide either to use adaptive cruise control (ACC) or a cooperative adaptive cruise control (CACC) scheme for driving. Specifically, this study explores the influences of ADS market-penetration and platoon properties on the overall performance of the mixed traffic stream. It is confirmed from the simulation that the grouping of ADS vehicles using a CACC system provides maximum mobility benefits and environmental improvements at the cost of reduced safety. It is proposed that, for a comprehensive balance in mobility, safety, and environmental advantages the platoon configuration should be adjusted dynamically considering the ADS market penetrations in the mixed traffic.

Article: Modified Traffic Flow Model with Connected Vehicle Microscopic Data for Proactive Variable Speed Limit Control.

The variable speed limit (VSL) technique using model predictive control (MPC) framework has the potential to improve the traffic flows on the freeways. However, the MPC framework directly relies on the traffic state prediction model in deciding the optimal speed of the traffic. This paper extends a VSL technique based on an MPC framework by incorporating microscopic online data for better prediction of the traffic flows using METANET macroscopic model. Simulations conducted on the VISSIM platform confirm that the proposed method improves the speed prediction accuracy, and hence improves mobility performance.

Article: Modeling and Prediction of Ride-Sharing Utilization Dynamics.

An efficient ride-sharing scheme may significantly reduce traffic congestion and facilitate better transportation services to realize smart cities. This paper analyzes a dataset of over 14 million taxi trips taken in New York City and proposes a network-centric approach for modeling and forecasting the potential ride-sharing utilization over time. Despite significant volatility of ride-sharing utilization, using the proposed approach the potential utilization can be forecasted reliably a few hours ahead of time.

\section{Traffic Light Control Considering CAVs}

Article: Context-Aware Intelligent Traffic Light Control through Secure Messaging.

This paper presents a secure messaging method for vehicle-to-infrastructure (V2I) communication, and based on it a traffic light control scheme has been developed. Using computationally lightweight protocol, the privacy of individual vehicles is maintained among themselves, while the identity of emergency vehicles and public transportation is securely received by the trusted controller of traffic lights. Compared to the existing traffic light control schemes, the proposed traffic responsive signal control scheme-adaptive Webster's method-significantly reduces the waiting time of vehicles in both light and heavy traffic conditions.

Article: A Separation Strategy for Connected and Automated Vehicles: Utilizing Traffic Light Information for Reducing Idling at Red Lights and Improving Fuel Economy.

Efficient flows of CAVs at a signalized intersection can be realized by forming platoons of suitable size. Within a platoon, all vehicles run through the intersection at the same speed and hence their trajectories are parallel to each other. However, for passing through successive intersections, the same platoon may not be able to pass through the next intersection fully in the given green signal. In this paper, a scheme for both velocity control and separation strategy of CAVs has been proposed that takes into account the traffic efficiency and fuel-saving simultaneously. Simulation results show that the proposed scheme improves both the travel time and fuel economy significantly on a typical urban road with successive signalized intersections.

\section{Traffic Monitoring Using CAVs}

Article: Potentialities of Autonomous Vehicles for Online Monitoring of Motorway Traffic.

Connectivity in vehicular traffic opens a new reliable way to collect traffic information online over a road-network, which may provide essential information for an effective control measure. Particularly, CAVs can be used as virtual sensors in estimating the traffic volumes on the road-ways. In this paper, based on the hypothesis that CAV traffic volume is a direct portion of total traffic volume, a new method of monitoring real-time traffic volume has been proposed. Furthermore, the capabilities of the proposed method are demonstrated using an experimental study based on vehicle navigation data available from smartphones. The developed method is found to be effective when the probe volume data are available at least with the penetration rate of 0.05 or higher. 


\title{
Conflicts of Interest
}

The editors declare that there are no conflicts of interest in publishing this editorial.

\author{
Md Abdus Samad Kamal \\ Mohsen Ramezani \\ Guoyuan Wu \\ Claudio Roncoli \\ Jackeline Rios-Torres \\ Olivier Orfila
}

\section{References}

[1] The World Bank, "Understanding poverty: transport," https:// www.worldbank.org/en/topic/transport.

[2] WHO, “Global status report on road safety," 2018, https://www. who.int/violence_injury_prevention/road_safety_status/2018/ en/.

[3] O. Orfila, D. Gruyer, K. Hamdi, and S. Glaser, "Safe and ecological speed profile planning algorithm for autonomous vehicles using a parametric multiobjective optimization procedure," International Journal ofAutomotive Engineering, vol. 10, no. 1, pp. 26-33, 2019.

[4] M. Ramezani and E. Ye, "Lane density optimisation of automated vehicles for highway congestion control," Transportmetrica $B$ : Transport Dynamics, vol. 7, no. 1, pp. 1096-1116, 2019.

[5] J. Rios-Torres and A. A. Malikopoulos, "A survey on the coordination of connected and automated vehicles at intersections and merging at highway on-ramps," IEEE Transactions on Intelligent Transportation Systems, vol. 18, no. 5, pp. 1066-1077, 2017.

[6] D. Tian, G. Wu, K. Boriboonsomsin, and M. J. Barth, "Performance measurement evaluation framework and cobenefit tradeoff analysis for connected and automated vehicles (CAV) applications: a survey," IEEE Intelligent Transportation Systems Magazine, vol. 10, no. 3, pp. 110-122, 2018.

[7] J. Rios-Torres and A. A. Malikopoulos, "Impact of partial penetrations of connected and automated vehicles on fuel consumption and traffic flow," IEEE Transactions on Intelligent Vehicles, vol. 3, no. 4, pp. 453-462, 2018.

[8] M. A. S. Kamal, T. Hayakawa, and J.-I. Imura, "Road-speed profile for enhanced perception of traffic conditions in a partially connected vehicle environment," IEEE Transactions on Vehicular Technology, vol. 67, no. 8, pp. 6824-6837, 2018.

[9] G. Perraki, C. Roncoli, I. Papamichail, and M Papageorgiou, "Evaluation of a model predictive control framework for motorway traffic involving conventional and automated vehicles," Transportation Research Part C: Emerging Technologies, vol. 92, pp. 456-471, 2018.

[10] Y. Ito, M. A. S. Kamal, T. Yoshimura, and S. Azuma, "Coordination of connected vehicles on merging roads using pseudo-perturbation-based broadcast control," IEEE Transactions on Intelligent Transportation Systems, vol. 20, no. 9, pp. 3496-3512, 2019. 


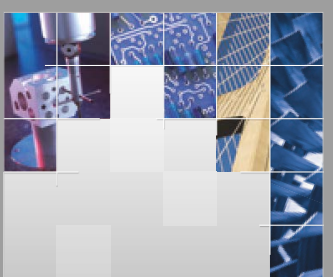

\section{Enfincering}
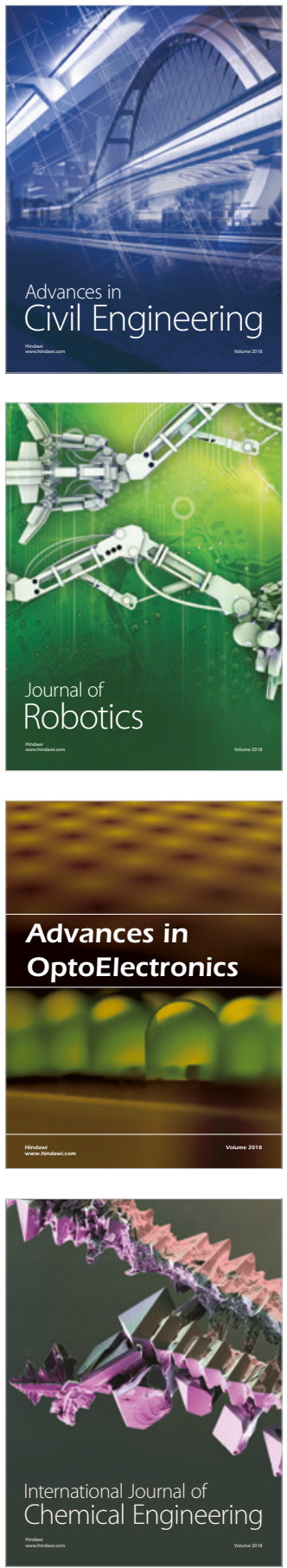

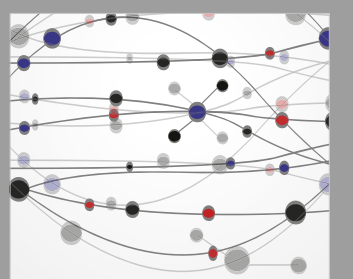

\section{Rotating \\ Machinery}

The Scientific World Journal

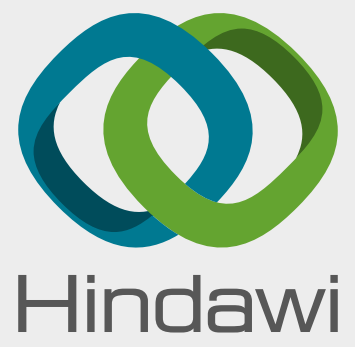

Submit your manuscripts at

www.hindawi.com
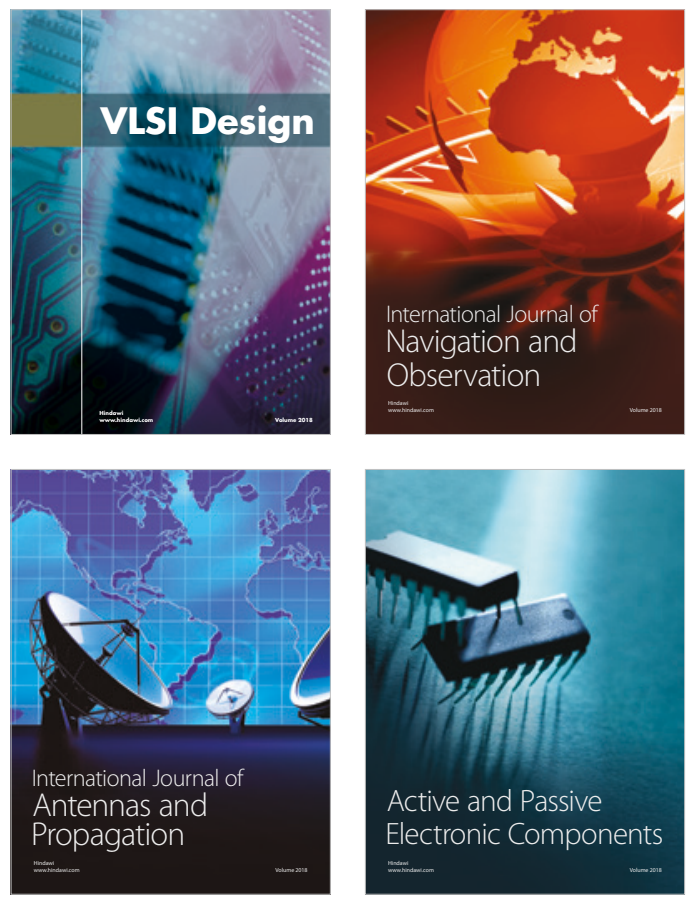
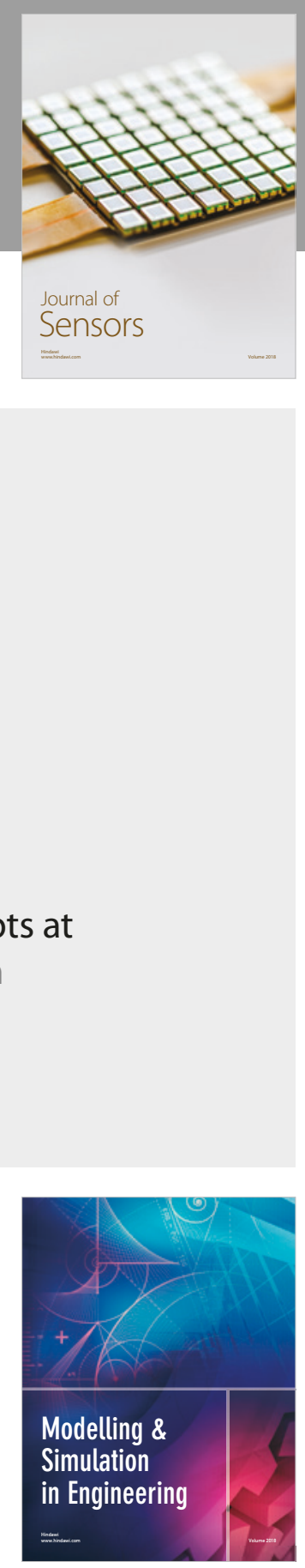

\section{Advances \\ Multimedia}
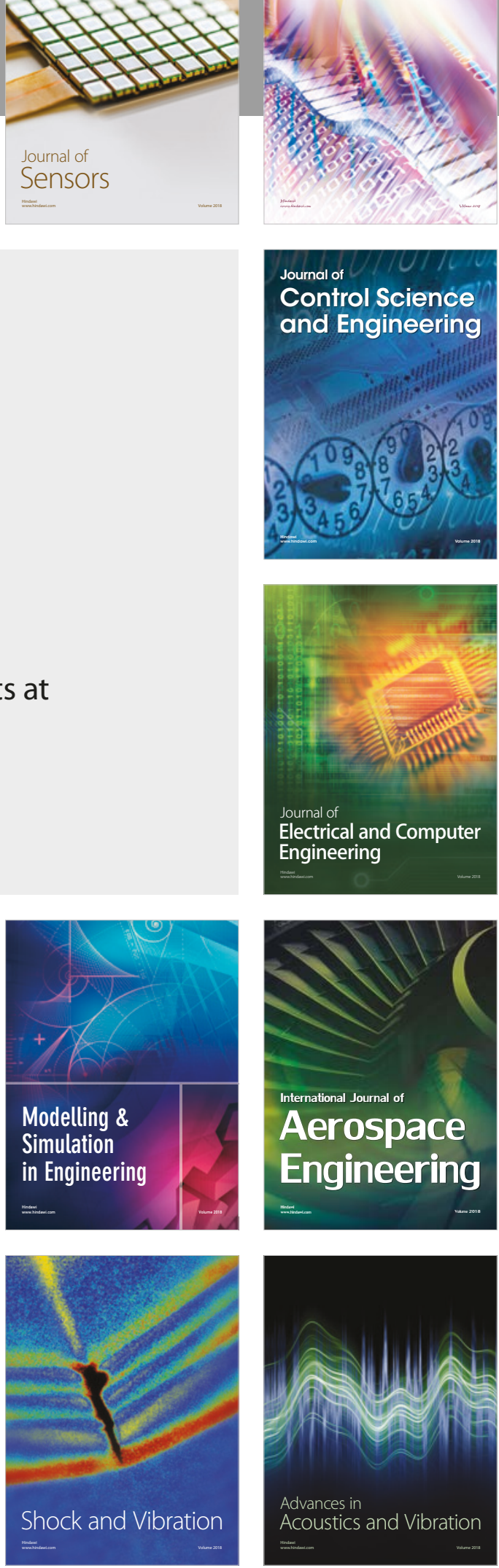